\title{
Effects of glucose tolerance on the changes provoked by glucose ingestion in microvascular function
}

\author{
A. Natali $\cdot$ S. Baldi $\cdot$ F. Vittone $\cdot$ E. Muscelli . \\ A. Casolaro • C. Morgantini • C. Palombo • \\ E. Ferrannini
}

Received: 2 August 2007 / Accepted: 25 January 2008 / Published online: 29 March 2008

(C) Springer-Verlag 2008

\begin{abstract}
Aims/hypothesis Hyperglycaemia and hyperinsulinaemia have opposite effects on endothelium-dependent vasodilatation in microcirculation, but the net effect elicited by glucose ingestion and the separate influence of glucose tolerance are unknown.

Methods In participants with normal glucose tolerance (NGT), impaired glucose tolerance (IGT) or diabetic glucose tolerance, multiple plasma markers of both oxidative stress and endothelial activation, and forearm vascular responses (plethysmography) to intra-arterial acetylcholine (ACh) and sodium nitroprusside (SNP) infusions were measured before and after glucose ingestion. In another IGT group, we evaluated the time-course of the skin vascular responses (laser Doppler) to ACh and SNP (by iontophoresis) 1, 2 and $3 \mathrm{~h}$ into the OGTT; the plasma glucose profile was then reproduced by means of a variable intravenous glucose infusion and the vascular measurements repeated.

Results Following oral glucose, plasma antioxidants were reduced by $5 \%$ to $10 \%(p<0.01)$ in all patient groups. The response to acetylcholine was not affected by glucose ingestion in any group, while the response to SNP was attenuated, particularly in the IGT group. The ACh:SNP ratio was slightly improved therefore in all groups, even in diabetic participants, in whom it was impaired basally. A time-dependent improvement in $\mathrm{ACh}: \mathrm{SNP}$ ratio was also
\end{abstract}

A. Natali $(\bowtie) \cdot$ S. Baldi $\cdot$ F. Vittone $\cdot$ E. Muscelli $\cdot$ A. Casolaro

C. Morgantini $\cdot$ C. Palombo $\cdot$ E. Ferrannini

Department of Internal Medicine, University of Pisa,

Via Roma, 67,

Pisa 56100, Italy

e-mail: anatali@ifc.cnr.it observed in skin microcirculation following oral glucose; this improvement was blunted when matched hyperglycaemia was coupled with lower hyperinsulinaemia (intravenous glucose).

Conclusions/interpretation Regardless of glucose tolerance, oral glucose does not impair endothelium-dependent vasodilatation either in resistance arteries or in the microcirculation, despite causing increased oxidative stress; the endogenous insulin response is probably responsible for countering any inhibitory effect on vascular function.

Keywords Endothelium - Glucose intolerance . Hyperglycaemia $\cdot$ Hyperinsulinaemia $\cdot$ Microcirculation . Nitric oxide - Oxidative stress $\cdot$ Resistance arteries .

Type 2 diabetes · Vascular function

$\begin{array}{ll}\text { Abbreviations } \\ \text { ACh } & \begin{array}{l}\text { acetylcholine } \\ \text { reactive oxygen metabolites }\end{array} \\ \text { EDV } & \begin{array}{l}\text { endothelium-dependent vasodilatation } \\ \text { ferric-reducing ability of plasma }\end{array} \\ \text { FRAP } & \text { soluble intracellular adhesion molecules } \\ \text { ICAM-1 } & \text { impaired glucose tolerance } \\ \text { IGT } & \text { normal glucose tolerance } \\ \text { NGT } & \text { microvascular endothelium-dependent va- } \\ \text { MV-EDV } & \text { sodilatation } \\ & \text { nitric oxide } \\ \text { NO } & \text { resistance arteries endothelium-dependent } \\ \text { RA-EDV } & \text { vasodilatation } \\ & \text { sodium nitroprusside } \\ \text { SNP } & \text { total antioxidant status } \\ \text { TAS } & \text { soluble vascular adhesion molecules } \\ \text { VCAM-1 } & \text { 8-iso-prostaglandin } \mathrm{F}_{2 \alpha}\end{array}$




\section{Introduction}

Clinical manifestations of atherosclerosis are more frequent in patients with type 2 diabetes than in the non-diabetic population [1] and largely contribute to reduced life expectancy and quality of life in these patients. This excess atherosclerosis is only partially explained by traditional risk factors [2]. Therefore, a role for hyperglycaemia itself emerges forcefully. The benefit of improved metabolic control on vascular complications is typically smaller than expected [3], suggesting that other non-traditional, diabetesrelated risk factors are involved and/or that glycaemic control is generally insufficient. Experimental evidence indicates that atherogenic mechanisms, such as endothelial dysfunction, oxidative stress and inflammation, may be chronically activated in diabetes [4-7], at least in part as a consequence of exaggerated postprandial glucose excursions [8-10]. The negative impact of hyperglycaemia-induced oxidative stress on endothelial dysfunction has been reported in a number of in vitro and animal studies [11-15]. Most of these studies, however, have applied rather extreme experimental conditions, making it difficult to assess their relevance either to diabetes, or to impaired glucose tolerance (IGT) where glucose excursions are smaller, smoother and associated with various degrees of hyperinsulinaemia.

With regard to the acute effect of hyperglycaemia on endothelium-dependent vasodilatation (EDV), conflicting results have been reported in vivo in humans, with some describing a neutral or a very mild and short-lasting effect [16-21] and others a negative impact [22-26]. Part of this heterogeneity can be ascribed to differences in the vascular district investigated (large, small or micro-vessels), the experimental technique adopted and the stimulus applied. Thus, isolated hyperglycaemia whether systemic (glucose clamp + somatostatin) or local (perfused forearm), hyperglycaemia with hyperinsulinaemia (oral glucose) or hyperglycaemia in combination with hyperlipidaemia (meal test) in participants with different degrees of glucose tolerance have all been tested. For small vessels (resistance arteries and the microcirculation), the balance of evidence indicates that while hyperglycaemia has a neutral $[16,21]$ or only a modest negative effect on EDV [5], hyperinsulinaemia exerts a consistent positive action [27-29]. The question of how glucose tolerance status may modulate the vascular response to physiological combinations of hyperinsulinaemia and hyperglycaemia has not been examined.

We hypothesised that in small vessels the net effect on EDV may depend on the relative intensity of hyperglycaemia and hyperinsulinaemia. To test this hypothesis, we measured EDV of resistance (forearm) arteries, i.e. resistance arteries endothelium-dependent vasodilatation (RA-EDV) before and after a standard OGTT in participants with normal glucose tolerance (NGT), IGT or type 2 diabetes, in whom different degrees of hyperglycaemia and hyperinsulinaemia are elicited by glucose ingestion. In another group of participants with IGT, we also evaluated whether glucose ingestion affected microvascular (skin) reactivity, i.e. microvascular endothelium-dependent vasodilatation (MV-EDV). Finally, we tested whether matched plasma glucose excursions coupled with smaller insulin increments (achieved with intravenous glucose infusions) revealed an imbalance between the two opposing forces. In all experiments, we also measured a large panel of plasma indices estimating the balance between oxidants and antioxidants.

\section{Methods}

Study participants For the RA-EDV study, 53 participants of either sex were selected from the outpatient clinic according to the following criteria: 40 to 60 years of age, BMI 25 to $35 \mathrm{~kg} / \mathrm{m}^{2}$, blood pressure $<150 / 90 \mathrm{mmHg}$, serum total cholesterol $<6.5 \mathrm{mmol} / 1$ and serum triacylglycerol $<3.4 \mathrm{mmol} / \mathrm{l}$. Exclusion criteria were acute or chronic inflammatory diseases, impairment in cardiac, liver or kidney function, endocrine abnormalities, current smoking or treatment with antioxidants. Participants who were receiving treatment with drugs known to have a strong influence on endothelial function (statins, dihydropyridine calcium-channel blockers and beta blockers) were excluded and any other drug treatment was withheld in all participants at least $24 \mathrm{~h}$ prior to the OGTT. Participants were classified as NGT, IGT or type 2 diabetes on the basis of their OGTT results.

For the MV-EDV study, a separate group of 15 IGT participants was recruited using the same criteria described above.

The study was approved by the local ethics committee and all participants signed a written consent form, in which all the procedures and their risk were described in detail.

Study design, RA-EDV All participants underwent a $75 \mathrm{~g}$ OGTT with timed measurements of plasma glucose and insulin concentrations. Before and at $120 \mathrm{~min}$ after glucose ingestion, the intra-arterial plasma concentration of the following markers of endothelial dysfunction and oxidative stress were measured: soluble intracellular adhesion molecules (ICAM-1) and vascular adhesion molecules (VCAM-1), reactive oxygen metabolites (dROMs), lipid hydroperoxides, malonyldialdehyde, ferric-reducing ability of plasma (FRAP), total antioxidant status (TAS) and ex vivo LDL-cholesterol resistance to copper-induced oxidation. In a subset of patients from each study group (ten NGT, 11 IGT and ten type 2 diabetic patients), RA-EDV in the forearm vasculature was measured (see below) before glucose ingestion (from time -90 to $0 \mathrm{~min}$ ) and after glucose ingestion (from 90 to 
$180 \mathrm{~min}$ ). The clinical characteristics of this subgroup did not differ from those of the whole study group.

Study design, $M V$-EDV All participants received an OGTT as described above. Skin microvascular reactivity (see below) was measured before and at 60,120 and $180 \mathrm{~min}$ after glucose ingestion. In nine participants, the study was repeated 1 to 2 weeks later using a variable intravenous glucose infusion to reproduce the individual plasma glucose time-course observed during the OGTT. For this 'isoglycaemic' protocol, plasma glucose was determined at the bedside every 5 to $10 \mathrm{~min}$ using a glucose analyser (Glucose Analyzer II; Beckman Instruments, Fullerton, CA, USA) and the glucose infusion was adjusted using an ad hoc-designed computer program.

Vascular procedures, $R A-E D V$ Vascular reactivity in resistance arteries was evaluated by the perfused forearm technique. Briefly, a Teflon cannula was inserted percutaneously into the brachial artery of the non-dominant arm under local anaesthesia ( $2 \%$ lidocaine) and used for drug infusion, blood sampling and blood pressure recordings. Forearm blood flow was measured by venous occlusion strain-gauge plethysmography (EC4; Hokanson, Bellevue, WA, USA); this was done three to six times at baseline and at the end of each of four $5 \mathrm{~min}$ steps of intra-arterial infusion of acetylcholine $(0.2$, $0.6,3.0$ and $12.0 \mu \mathrm{g} \mathrm{min}^{-1} 100 \mathrm{ml}^{-1}$ of forearm tissue) and sodium nitroprusside (SNP; 0.05, 0.20, 0.80 and $2.40 \mu \mathrm{g}$ $\min ^{-1} 100 \mathrm{ml}^{-1}$ ). Forearm volume was measured from the water displacement induced by immersion of the forearm into a graduated cylinder. To control for the intra- and intertest variability in blood pressure and basal blood flow, forearm vascular conductance was calculated as the ratio $(\times 100)$ of blood flow to the simultaneously measured mean blood pressure; the per cent change of this ratio with respect to baseline was used as the primary outcome variable.

Vascular procedures, $M V-E D V$ Peripheral microvascular function was assessed with a laser Doppler flowmeter (Periflux PF4001, standard probes PF408; Perimed, Järfälla Sweden), which provides real-time measurements of skin blood flow, coupled with iontophoresis, which allows subcutaneous delivery of vasoactive drugs. Skin blood flow measurements were performed at controlled room temperature, with the participant in a supine position. Laser Doppler probes were positioned at corresponding sites of the volar aspect of each of the two forearms after finding a stable basal flow trace. After 10 min of baseline recording, acetylcholine (1\% solution in saline; Farmigea, Pisa, Italy) and SNP (1\% solution in saline; Malesci, Florence, Italy) were applied simultaneously in both probes by iontophoresis in five consecutive doses $(0.10 \mathrm{~mA} \times 20 \mathrm{~s}$ and $0.06 \mathrm{~mA} \times$ $20 \mathrm{~s}$ per dose of acetylcholine and SNP, respectively). To avoid the non-specific vasodilatation that is observed when a negative current (necessary for SNP delivery) is applied to the skin, we used low-intensity voltages and local pretreatment with a lidocaine cream (Emla; AstraZeneca, Milan, Italy) $5 \mathrm{~min}$ before iontophoresis. To control for variability on baseline measures, each microvascular response was expressed as the per cent change from the baseline.

Assays Plasma insulin was measured by radioimmunoassay (InsKit; Linco Research, St. Charles, MO, USA). Spectrophotometry was employed to measure dROMs (dROMs Test; Diacron, Grosseto, Italy), TAS (TAS Test; Randox, Oceanside, CA, USA), FRAP (according to the method of Benzie et al. [30]), lipid hydroperoxides (by the method of Wolff [31]) and malonyldialdehyde (LPO-586; Oxis International, Foster City, CA, USA). Total plasma isoprostane 8-iso-prostaglandin $\mathrm{F}_{2 \alpha}\left(8\right.$-iso- $\mathrm{PGF}_{2 \alpha}$ ) levels were assayed using a commercially available enzyme immunoassay (Assay Designs, Ann Arbour, MI, USA) on samples to which BHT was added before storage at $-80^{\circ} \mathrm{C}$. Ex vivo LDL oxidisability was determined as the lag-phase of conjugated diene formation upon incubation with copper sulphate (at concentrations of 0.25 and $1.0 \mu \mathrm{mol} / \mathrm{l}$ ), as previously described [32]. ICAM-1 and VCAM-1 concentrations were measured using commercially available immunoenzymatic assays (human sVCAM-1 and human sICAM-1; Biosource International, Camarillo, CA, USA).

Statistical analysis Group differences were tested by $\chi^{2}$, ANOVA or the Kruskal-Wallis test, as appropriate. Changes from baseline were tested by paired $t$ test or twoway ANOVA for repeated measures. A value of $p \leq 0.05$ was considered as significant. Given this sample size (ten per group) and the inter-individual variability (35\%) of the vascular measurements expressed as maximal per cent increment above baseline (data from our laboratory), we calculated that this study had the power ( 0.9 at an alpha value of 0.01 ) to detect changes caused by glucose ingestion (with respect to fasting) equal or greater than $20 \%$.

\section{Results}

RA-EDV study Age, BMI, arterial blood pressure and serum lipids differed across groups as expected from glucose tolerance status (Table 1). Plasma glucose and insulin concentrations showed the characteristic rise in response to glucose ingestion (Fig. 1). The plasma glucose gradient between baseline and the last $90 \mathrm{~min}$ of the OGTT (when RA-EDV was measured) averaged $1.26 \pm 0.18 \mathrm{mmol} / \mathrm{l}$ in the NGT group and was twofold and 3.5-fold higher in IGT and diabetic participants, respectively ( $p<0.01$ for both). Fast- 
Table 1 Characteristics of the study participants

\begin{tabular}{lllll}
\hline & NGT & IGT & Diabetes & $p$ value* \\
\hline$N$ & 20 & 16 & 17 & \\
Sex (women/men) $(n)$ & $6 / 14$ & $6 / 10$ & $5 / 12$ & NS \\
Age (years) & $49 \pm 2$ & $52 \pm 2$ & $58 \pm 2$ & $<0.01$ \\
BMI (kg/m $)$ & $27.9 \pm 0.9$ & $29.5 \pm 1.2$ & $29.3 \pm 1.0$ & NS \\
SBP (mmHg) & $129 \pm 3$ & $122 \pm 3$ & $139 \pm 4$ & $<0.01$ \\
DBP (mmHg) & $78 \pm 2$ & $79 \pm 2$ & $81 \pm 2$ & NS \\
HT (yes/no) $(n)$ & $4 / 16$ & $3 / 13$ & $5 / 12$ & NS \\
HbA $(\%)$ & $5.6 \pm 0.1$ & $5.9 \pm 0.3$ & $7.2 \pm 0.5$ & $<0.01$ \\
Total chol (mmol/l) & $4.87 \pm 0.21$ & $5.61 \pm 0.34$ & $4.89 \pm 0.29$ & NS \\
LDL-chol (mmol/l) & $3.32 \pm 0.18$ & $3.68 \pm 0.32$ & $3.29 \pm 0.26$ & NS \\
HDL-chol (mmol/l) & $1.16 \pm 0.05$ & $1.32 \pm 0.13$ & $0.95 \pm 0.08$ & $<0.05$ \\
Triacylglycerol (mmol/l) & $1.08 \pm 0.12$ & $1.33 \pm 0.15$ & $1.45 \pm 0.10$ & 0.05 \\
Fasting NEFA (mmol/l) & $0.52 \pm 0.03$ & $0.60 \pm 0.04$ & $0.55 \pm 0.03$ & NS \\
OGTT-NEFA (mmol/l) & $0.15 \pm 0.02$ & $0.14 \pm 0.03$ & $0.21 \pm 0.03$ & $<0.01$ \\
\hline
\end{tabular}

ing plasma insulin concentrations were similar across groups; mean values over the last $90 \mathrm{~min}$ of the OGTT were $379 \pm 30,552 \pm 75$ and $432 \pm 54 \mathrm{pmol} / \mathrm{l}$ in the NGT, IGT and diabetes groups, respectively; the corresponding increment above baseline was $50 \%$ greater in the IGT than in the NGT and diabetes groups ( $p<0.05$ for both).

Of the circulating markers, only fasting VCAM-1 and TAS levels were significantly different according to glucose tolerance status. Plasma 8-iso- $\mathrm{PGF}_{2 \alpha}$ levels were markedly and similarly elevated in both IGT and diabetic participants $(p<0.05$ with respect to NGT). Following glucose ingestion, plasma concentrations of ICAM-1, dROMs, lipid hydroperoxides and malonyldialdehyde, as well as the lag-phase of copper-induced LDL oxidation did not change significantly in any of the groups, whereas VCAM-1, TAS and FRAP showed small reductions $(-6,-6$ and $-9 \%$, respectively, $p<0.05$ for all), which were quantitatively similar across groups (Table 2). Plasma 8 -iso- $\mathrm{PGF}_{2 \alpha}$ showed a tendency to increase in response to glucose ingestion; however, due to the large inter-individual variability, this increase did not achieve statistical significance, either when each group was analysed separately (Mann-Whitney test), or when all participants were considered together $(p=0.09$ by ANOVA for repeated measure).

Pre-test-infusion forearm blood flow rates and haemodynamic parameters were similar across groups and relatively stable throughout the study (Table 3). At the maximal infusion rates of $\mathrm{ACh}$ and SNP, small (5\% and $10 \%$ respectively) but consistent ( $p<0.05$ for both) declines in blood pressure coupled with a modest increase in heart rate were observed in all groups, both under fasting conditions and during the OGTT, thereby justifying the use of conductance as a measure of local vasodilation.

Under fasting conditions, the vascular response to $\mathrm{ACh}$ was blunted in individuals with type 2 diabetes $(p<0.05$ vs
NGT and IGT), while the response to SNP was similar in the three groups (Fig. 2). After glucose ingestion, AChinduced vasodilatation was similar to the response observed under fasting conditions in all groups; the response to SNP in NGT and diabetic participants was also unaffected by glucose ingestion, whereas it was modestly depressed in the IGT group $(p<0.04)$. In the fasting state as well as after glucose ingestion, the ratio of $\mathrm{ACh}$ to SNP maximal responses (ACh:SNP) was reduced only in individuals with type 2 diabetes $(p<0.05$ vs IGT and NGT). During the OGTT, the ACh:SNP ratio slightly increased in all groups (from $1.47 \pm 0.12$ to $1.55 \pm 0.21,1.51 \pm 0.30$ to $1.95 \pm 0.38$ and $1.01 \pm 0.13$ to $1.38 \pm 0.24$ in NGT, IGT and diabetic participants respectively, $p<0.05$ for glucose tolerance and OGTT effects by ANOVA for repeated measures).

$M V-E D V$ study The IGT patients undergoing the MV-EDV study had similar clinical characteristics to the IGT group undergoing the RA-EDV study (data not shown). Skin perfusion showed an eightfold increase in maximal response to local $\mathrm{ACh}$ and a fivefold increment in maximal response to SNP. At $2 \mathrm{~h}$ into the OGTT, no change in the response to $\mathrm{ACh}$ and an attenuation of the response to SNP $(p<0.05)$ were observed (Fig. 3). The time-course showed that the response to $\mathrm{ACh}$ declined at $60 \mathrm{~min}(p<0.05)$ and recovered thereafter, while the response to SNP was progressively attenuated over time ( $p<0.05$; Fig. 4). Consequently, the ACh:SNP ratio showed some increase at $120 \mathrm{~min}$ into the OGTT $(p=0.09)$.

In the participants undergoing the isoglycaemic experiment, the plasma glucose profile seen with oral glucose was closely matched by the variable intravenous glucose infusion. As expected, the increase in plasma insulin concentrations was markedly ( $\sim 50 \%)$ smaller (Fig. 5). The time-course of the ACh:SNP ratio in the group of 

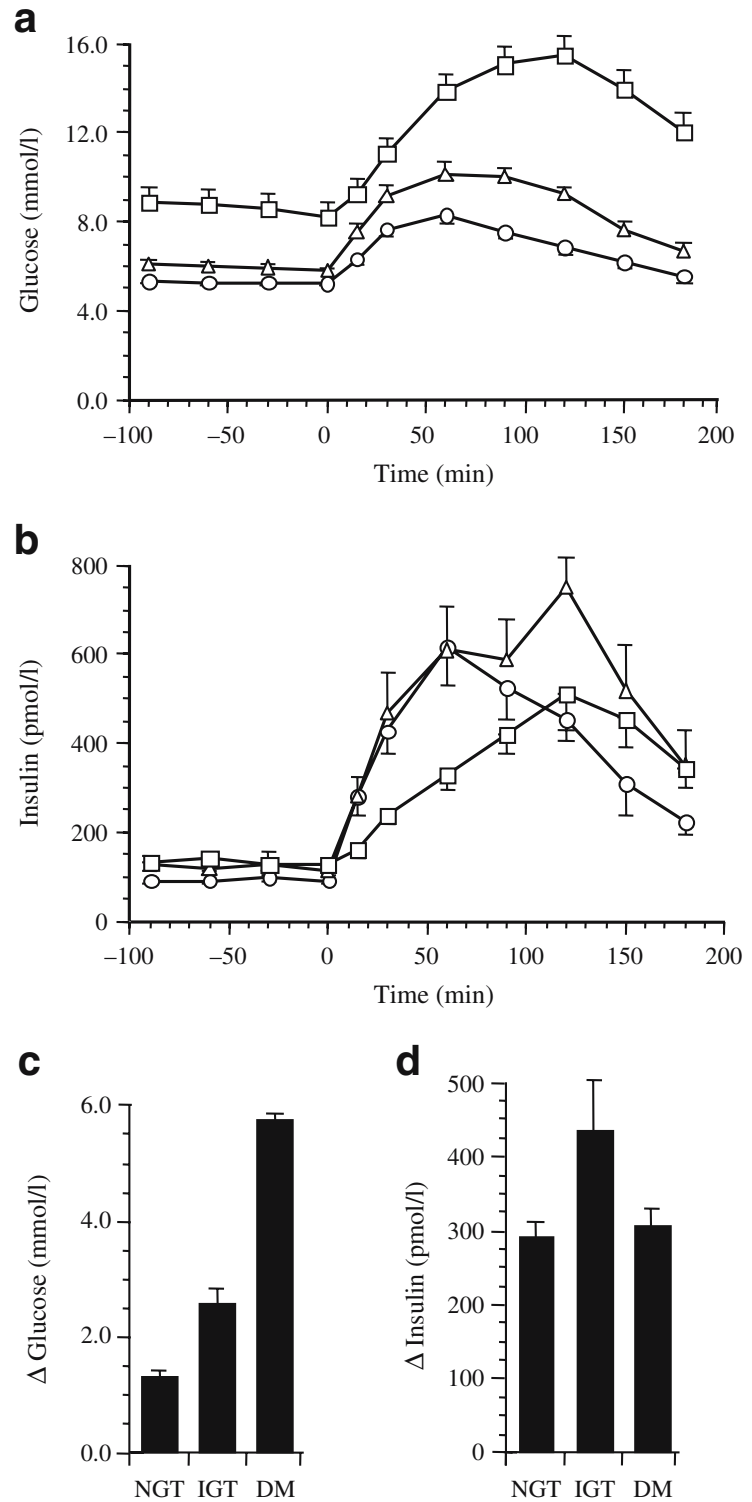

Fig. 1 Time-course of plasma glucose (a) and insulin (b) responses to oral glucose administration in NGT (circle), IGT (triangle) and diabetic (DM; square) individuals. The corresponding mean glucose (c) and insulin (d) increments above basal are shown in the bar graphs. Fasting phase, -100 to $0 \mathrm{~min}$; OGTT phase, 0 to $200 \mathrm{~min}$

participants undergoing the two tests showed that the only difference between the two experimental conditions was the response at $120 \mathrm{~min}$, at which time the improvement seen with oral glucose was not observed during intravenous glucose administration $(p<0.05)$.

\section{Discussion}

In participants with any degree of glucose tolerance, the acute hyperglycaemia and hyperinsulinaemia elicited by glucose ingestion induced a mild oxidative stress, as reflected in the reduction of circulating antioxidants and the tendency of plasma 8 -iso- $\mathrm{PGF}_{2 \alpha}$ to increase. Such stress, however, did not impair EDV in resistance arteries or the microcirculation, did not modify LDL resistance to in vitro oxidation and did not increase the plasma concentration of vascular adhesion molecules. Indeed, the ratio of acetylcholine-mediated dilatation to SNP-mediated dilata-

Table 2 Markers of oxidative stress and endothelial dysfunction

\begin{tabular}{|c|c|c|c|}
\hline & NGT & IGT & Diabetes \\
\hline \multicolumn{4}{|c|}{ ICAM-1 (ng/ml) } \\
\hline Fasting & $323 \pm 38$ & $263 \pm 42$ & $301 \pm 42$ \\
\hline OGTT & $305 \pm 28$ & $244 \pm 36$ & $291 \pm 45$ \\
\hline$\Delta$ & $-25 \pm 17$ & $-20 \pm 25$ & $-7 \pm 11$ \\
\hline \multicolumn{4}{|c|}{ VCAM (ng/ml) } \\
\hline Fasting & $551 \pm 61$ & $683 \pm 61$ & $792 \pm 118^{*}$ \\
\hline OGTT & $509 \pm 59$ & $601 \pm 61$ & $768 \pm 89$ \\
\hline$\Delta^{\dagger}$ & $-48 \pm 54$ & $-76 \pm 28$ & $-24 \pm 60$ \\
\hline \multicolumn{4}{|c|}{ dROMS (U/1) } \\
\hline Fasting & $173 \pm 11$ & $182 \pm 23$ & $172 \pm 23$ \\
\hline OGTT & $170 \pm 17$ & $182 \pm 25$ & $164 \pm 21$ \\
\hline$\Delta$ & $-3 \pm 11$ & $0 \pm 19$ & $-9 \pm 8$ \\
\hline \multicolumn{4}{|c|}{$\mathrm{LOOH}(\mu \mathrm{mol} / \mathrm{l})$} \\
\hline Fasting & $4.8 \pm 1.0$ & $7.9 \pm 2.1$ & $7.4 \pm 1.8$ \\
\hline OGTT & $6.4 \pm 1.2$ & $8.6 \pm 2.0$ & $7.1 \pm 1.8$ \\
\hline$\Delta$ & $1.6 \pm 0.8$ & $0.7 \pm 1.3$ & $-0.2 \pm 1.3$ \\
\hline \multicolumn{4}{|c|}{ MDA $(\mu \mathrm{mol} / 1)$} \\
\hline Fasting & $5.9 \pm 2.0$ & $7.5 \pm 1.0$ & $8.3 \pm 1.9$ \\
\hline OGTT & $6.7 \pm 2.4$ & $7.0 \pm 0.9$ & $8.5 \pm 1.7$ \\
\hline$\Delta$ & $0.7 \pm 0.6$ & $-0.4 \pm 0.7$ & $0.3 \pm 0.6$ \\
\hline \multicolumn{4}{|c|}{ 8-iso-PGF $2 \alpha(\mathrm{pg} / \mathrm{ml})$} \\
\hline Fasting & $1,673 \pm 567$ & $6,120 \pm 893$ & $5,223 \pm 1,633^{*}$ \\
\hline OGTT & $2,363 \pm 948$ & $7,139 \pm 846$ & $6,717 \pm 1,906$ \\
\hline$\Delta$ & $690 \pm 429$ & $1,018 \pm 1,200$ & $1,492 \pm 1,211$ \\
\hline \multicolumn{4}{|c|}{ Lag-phase 0.25 (min) } \\
\hline Fasting & $166 \pm 4$ & $166 \pm 7$ & $159 \pm 5$ \\
\hline OGTT & $169 \pm 6$ & $158 \pm 6$ & $156 \pm 4$ \\
\hline$\Delta$ & $3 \pm 3$ & $-6 \pm 3$ & $-3 \pm 3$ \\
\hline \multicolumn{4}{|c|}{ Lag-phase 1.0 (min) } \\
\hline Fasting & $70 \pm 2$ & $69 \pm 2$ & $67 \pm 2$ \\
\hline OGTT & $69 \pm 2$ & $68 \pm 2$ & $67 \pm 2$ \\
\hline$\Delta$ & $1 \pm 2$ & $-1 \pm 1$ & $-1 \pm 1$ \\
\hline \multicolumn{4}{|c|}{ TAS $(\mu \mathrm{mol} / 1)$} \\
\hline Fasting & $893 \pm 83$ & $1,031 \pm 76$ & $1,001 \pm 47^{*}$ \\
\hline OGTT & $818 \pm 67$ & $959 \pm 75$ & $911 \pm 53$ \\
\hline$\Delta^{\dagger}$ & $-75 \pm 85$ & $-73 \pm 35$ & $-91 \pm 47$ \\
\hline \multicolumn{4}{|c|}{ FRAP $(\mu \mathrm{mol} / 1)$} \\
\hline Fasting & $722 \pm 36$ & $771 \pm 44$ & $770 \pm 39$ \\
\hline OGTT & $676 \pm 31$ & $735 \pm 44$ & $740 \pm 31$ \\
\hline$\Delta^{\dagger}$ & $-46 \pm 21$ & $-35 \pm 28$ & $-46 \pm 21$ \\
\hline
\end{tabular}

Values are means \pm SEM

OGTT was measured $2 \mathrm{~h}$ into the OGTT

$\Delta$, difference between OGTT and fasting value

LOOH, lipid hydroperoxides; MDA, malonyldialdehyde

$* p \leq 0.05$ across groups by Kruskal-Wallis; ${ }^{\dagger} p \leq 0.05$ OGTT vs fasting by ANOVA or Mann-Whitney 
Table 3 Haemodynamic data

Values are mean \pm SEM

bpm, beats per minute; FBF, forearm blood flow; FBFc, FBF in control (non-infused) forearm; HR, heart rate; MBP, mean blood pressure

\begin{tabular}{|c|c|c|c|c|c|c|}
\hline & \multicolumn{2}{|l|}{ NGT } & \multicolumn{2}{|l|}{ IGT } & \multicolumn{2}{|l|}{ Diabetes } \\
\hline & Fasting & OGTT & Fasting & OGTT & Fasting & OGTT \\
\hline \multicolumn{7}{|l|}{ Pre-acetylcholine } \\
\hline $\mathrm{FBF}\left(\mathrm{ml} \mathrm{min}{ }^{-1} 100 \mathrm{ml}^{-1}\right)$ & $1.9 \pm 0.2$ & $1.9 \pm 0.2$ & $2.6 \pm 0.4$ & $2.3 \pm 0.2$ & $2.5 \pm 0.4$ & $2.5 \pm 0.2$ \\
\hline $\mathrm{FBFc}\left(\mathrm{ml} \mathrm{min}{ }^{-1} 100 \mathrm{ml}^{-1}\right)$ & $2.1 \pm 0.3$ & $1.9 \pm 0.2$ & $2.4 \pm 0.4$ & $1.8 \pm 0.1$ & $2.5 \pm 0.3$ & $2.1 \pm 0.3$ \\
\hline MBP (mmHg) & $93 \pm 2$ & $90 \pm 2$ & $89 \pm 3$ & $86 \pm 3$ & $93 \pm 2$ & $90 \pm 3$ \\
\hline HR (bpm) & $61 \pm 3$ & $62 \pm 4$ & $65 \pm 3$ & $66 \pm 2$ & $69 \pm 2$ & $72 \pm 3$ \\
\hline \multicolumn{7}{|l|}{ Acetylcholine max } \\
\hline $\mathrm{FBF}\left(\mathrm{ml} \mathrm{min}{ }^{-1} 100 \mathrm{ml}^{-1}\right)$ & $21.6 \pm 2.7$ & $22.1 \pm 2.2$ & $23.5 \pm 2.6$ & $25.7 \pm 2.4$ & $18.5 \pm 1.3$ & $22.4 \pm 2.1$ \\
\hline $\operatorname{FBFc}\left(\mathrm{ml} \mathrm{min}{ }^{-1} 100 \mathrm{ml}^{-1}\right)$ & $2.3 \pm 0.3$ & $2.0 \pm 0.2$ & $2.5 \pm 0.4$ & $2.6 \pm 0.4$ & $2.5 \pm 0.4$ & $2.4 \pm 0.4$ \\
\hline $\operatorname{MBP}(\mathrm{mmHg})$ & $90 \pm 2$ & $88 \pm 2$ & $83 \pm 4$ & $84 \pm 3$ & $89 \pm 3$ & $91 \pm 3$ \\
\hline HR (bpm) & $63 \pm 4$ & $67 \pm 4$ & $65 \pm 3$ & $71 \pm 4$ & $69 \pm 3$ & $72 \pm 3$ \\
\hline \multicolumn{7}{|l|}{ Pre-SNP } \\
\hline $\mathrm{FBF}\left(\mathrm{ml} \mathrm{min}{ }^{-1} 100 \mathrm{ml}^{-1}\right)$ & $2.4 \pm 0.2$ & $2.7 \pm 0.3$ & $3.2 \pm 0.3$ & $3.3 \pm 04$ & $2.4 \pm 0.2$ & $2.7 \pm 0.3$ \\
\hline $\mathrm{FBFc}\left(\mathrm{ml} \mathrm{min}{ }^{-1} 100 \mathrm{ml}^{-1}\right)$ & $1.7 \pm 0.2$ & $1.8 \pm 0.2$ & $2.3 \pm 0.4$ & $2.0 \pm 0.2$ & $2.0 \pm 0.3$ & $2.2 \pm 0.3$ \\
\hline MBP (mmHg) & $95 \pm 2$ & $93 \pm 2$ & $87 \pm 2$ & $86 \pm 3$ & $92 \pm 3$ & $94 \pm 2$ \\
\hline HR (bpm) & $61 \pm 4$ & $68 \pm 5$ & $64 \pm 2$ & $67 \pm 3$ & $68 \pm 2$ & $67 \pm 2$ \\
\hline \multicolumn{7}{|l|}{ SNP max } \\
\hline $\mathrm{FBF}\left(\mathrm{ml} \mathrm{min}{ }^{-1} 100 \mathrm{ml}^{-1}\right)$ & $18.5 \pm 1.8$ & $20.0 \pm 2.4$ & $21.7 \pm 1.5$ & $19.6 \pm 1.5$ & $19.2 \pm 1.1$ & $20.2 \pm 2.4$ \\
\hline $\operatorname{FBFc}\left(\mathrm{ml} \mathrm{min}^{-1} 100 \mathrm{ml}^{-1}\right)$ & $1.9 \pm 0.2$ & $1.9 \pm 0.2$ & $2.7 \pm 0.4$ & $2.5 \pm 0.4$ & $2.4 \pm 0.3$ & $2.4 \pm 0.5$ \\
\hline MBP (mmHg) & $86 \pm 3$ & $87 \pm 2$ & $83 \pm 3$ & $82 \pm 3$ & $86 \pm 3$ & $89 \pm 3$ \\
\hline HR (bpm) & $70 \pm 5$ & $71 \pm 5$ & $73 \pm 4$ & $73 \pm 3$ & $71 \pm 3$ & $75 \pm 2$ \\
\hline
\end{tabular}

tion was consistently higher during glucose absorption than in the fasting state in all patient groups, suggesting that the overall effect of oral glucose ingestion on vascular reactivity was, if any, slightly favourable. Furthermore, this pattern of responses was essentially the same in the resistance arteries of the forearm and in the microvasculature of the skin, despite the structural and functional differences between these two regions.

Further information was provided by the difference in responses to acetylcholine and direct nitric oxide (NO) a

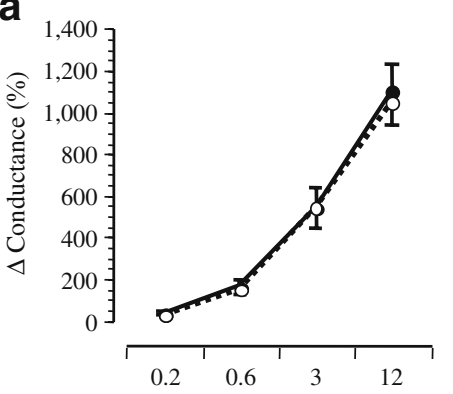

Drug infusion rate $\left(\mu \mathrm{g} \mathrm{min}{ }^{-1} 100 \mathrm{ml}^{-1}\right)$

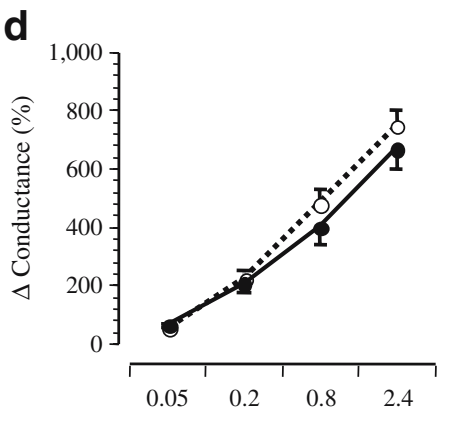

Drug infusion rate $\left(\mu \mathrm{g} \mathrm{min}{ }^{-1} 100 \mathrm{ml}^{-1}\right)$

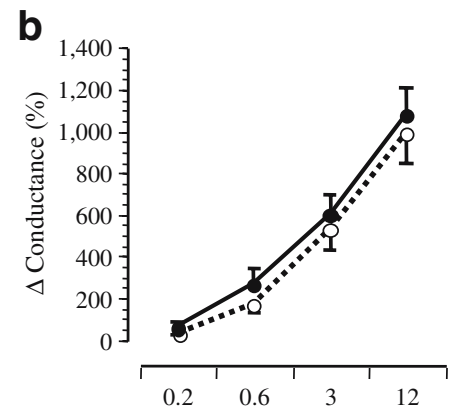

Drug infusion rate $\left(\mu \mathrm{g} \mathrm{min}{ }^{-1} 100 \mathrm{ml}^{-1}\right)$

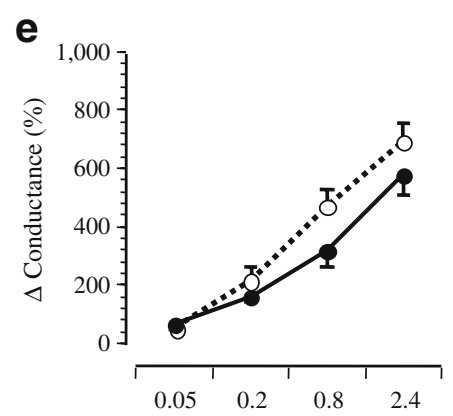

Drug infusion rate $\left(\mu \mathrm{g} \mathrm{min}{ }^{-1} 100 \mathrm{ml}^{-1}\right)$

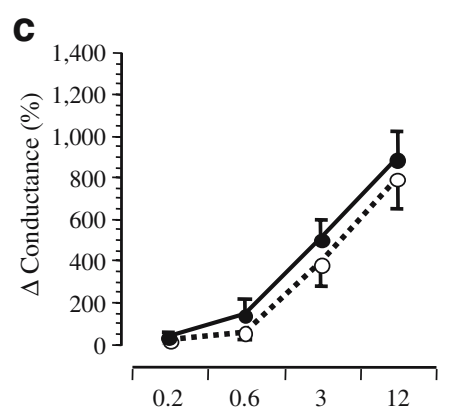

Drug infusion rate $\left(\mu \mathrm{g} \mathrm{min}{ }^{-1} 100 \mathrm{ml}^{-1}\right)$

f

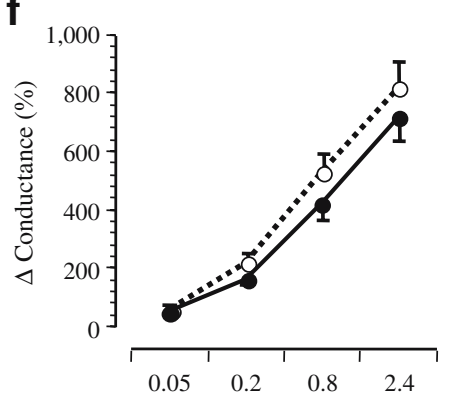

Drug infusion rate $\left(\mu \mathrm{g} \mathrm{min}{ }^{-1} 100 \mathrm{ml}^{-1}\right)$

Fig. 2 Forearm blood flow responses (as per cent changes in vascular conductance above baseline) to the graded intra-arterial infusion of acetylcholine $(\mathbf{a}-\mathbf{c})$ and $\operatorname{SNP}(\mathbf{d}-\mathbf{f})$ in $\operatorname{NGT}(\mathbf{a}, \mathbf{d})$, IGT $(\mathbf{b}, \mathbf{e})$ and

diabetic participants $(\mathbf{c}, \mathbf{f})$ in the fasting state (black circle, solid line) and during the final $90 \mathrm{~min}$ of a $3 \mathrm{~h}$ OGTT (white circle, dotted line) Data are mean \pm SEM. $p<0.014$ (e) 
a

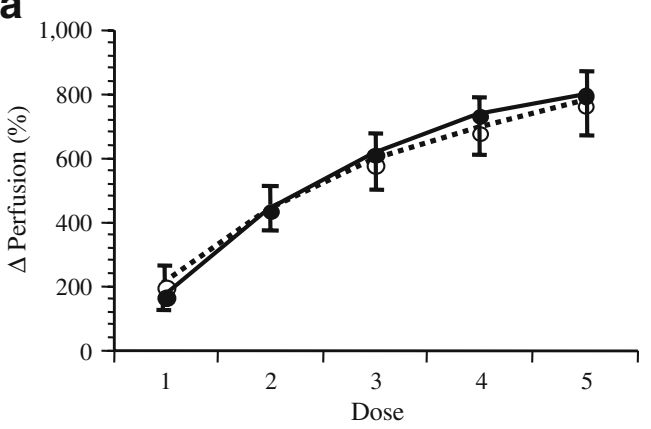

b

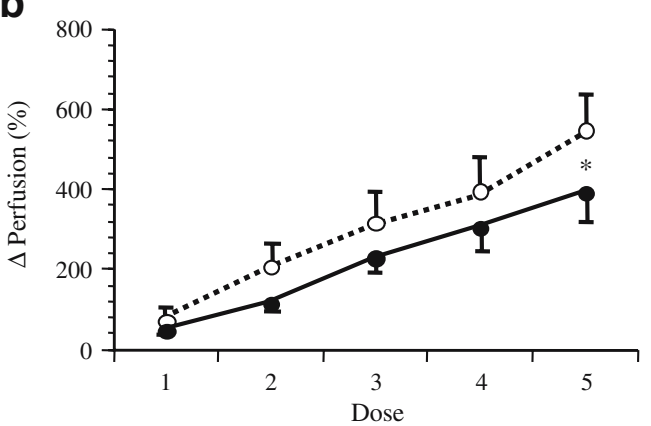

Fig. 3 Dose-responses to acetylcholine (a) and SNP (b) mediated vasodilatation in the skin microvasculature of IGT participants in the fasting state (black circle, solid line) and during the final $90 \mathrm{~min}$ of a 3 h OGTT (white circle, dotted lines). ${ }^{*} p<0.05$

supply (by SNP) and their time-course. In skin microvessels, the maximal response to $\mathrm{ACh}$ was only transiently reduced 1 $\mathrm{h}$ after glucose ingestion, in phase with the glycaemic peak; it recovered soon after (Fig. 4). In contrast, responses to SNP
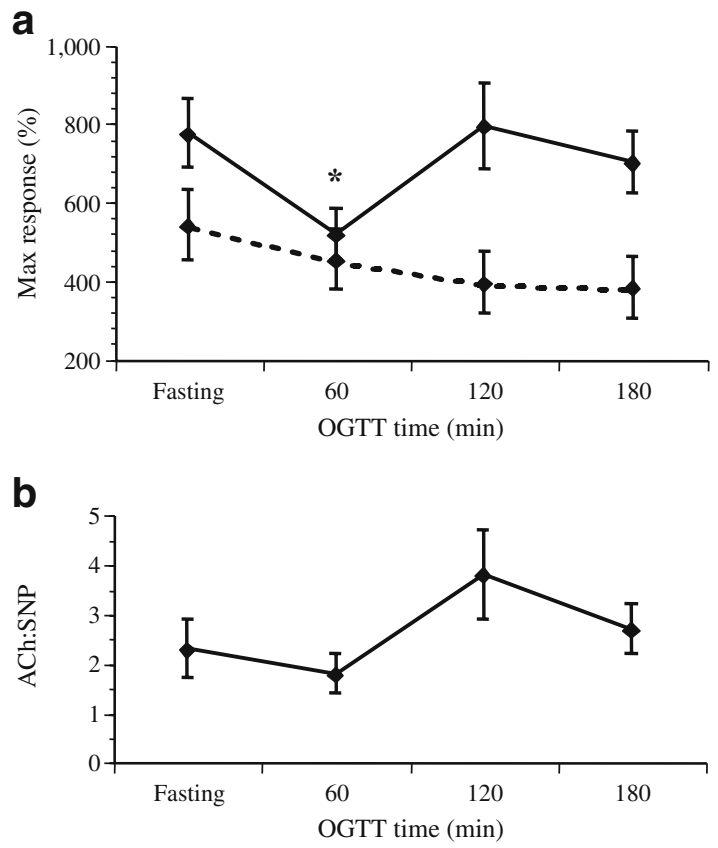

Fig. 4 Time-course of (a) maximal response to ACh (solid line) and SNP (dotted line). b Their ratio in the microvasculature of IGT participants before and during oral glucose administration. ${ }^{*} p<0.05$ tended to be lower after oral glucose than basally in resistance arteries (Fig. 2) and the skin (Fig. 3), and to decline over time in the skin subsequent to glucose ingestion (Fig. 4). When the glycaemic and insulinaemic stimuli were dissociated, as in the intravenous glucose experiment, the ACh:SNP ratio was significantly lower with intravenous than with oral glucose, but did not fall below its fasting value (Fig. 5). Therefore, we conclude that physiological (in size and time-course) changes in plasma glucose and insulin concentrations exert opposite effects on the EDV, with hyperinsulinaemia protecting against any inhibitory action of glucose loading.

These results are consistent with in vitro evidence showing that insulin stimulates NO release by endothelial cells [33] and with in vivo evidence showing that insulin potentiates acetylcholine-induced vasodilatation in resistance arteries [27] and in both skin [28] and muscle [29] microcirculation. This specific effect, however, appears attenuated in insulinresistant states [34], but we have previously shown that, in these conditions [27], insulin can still improve the vascular response to $\mathrm{ACh}$ exploiting its hyperpolarising effect. Alternatively, insulin might lose its positive effect on acetylcholine-mediated vasodilatation, which is mainly dependent on endothelial NO synthase activation, but not the ability to preserve the endothelial cell from the negative consequences of hyperglycaemia, which is known to act mainly through oxidative stress. In this regard, it is interesting to note that a chronic improvement in metabolic control did not modify the response to ACh, but restored the ability of insulin to potentiate the vascular response to $\mathrm{ACh}$ [35]. Alternatively, insulin might favour EDV by lowering plasma concentrations of NEFA, a substrate that negatively affects EDV [36]. Interestingly, in this context, the largest OGTT-induced NEFA gradient was observed in IGT participants: $76 \%$ vs $68 \%$ in NGT and $62 \%$ in diabetic participants $(p<0.01$ by Kruskal-Wallis; Table 1$)$.

Provided that we have evaluated only the spectrum of glucose intolerance from normal to mild diabetes, our results do not support the hypothesis that acute physiological hyperglycaemia causes significant deterioration of EDV. The well-established tenet that acute hyperglycaemia impairs endothelial function is mainly based on in vitro studies using pharmacological $(22 \mathrm{mmol} / \mathrm{l}$ or higher) concentrations of glucose $[12,13]$ without insulin or on ex vivo studies using experimental animal models characterised by severe hyperglycaemia [14, 15, 37, 38]. In humans, support for this notion derives mostly from studies using the OGTT as the stimulus, and flow-mediated dilatation of large arteries as the index of endothelial function [23-25]. Rather surprisingly, the results of these studies are not consistent $[17,19]$ despite the fact that hyperinsulinaemia itself has been claimed to have a selective, major negative effect on this segment of the 

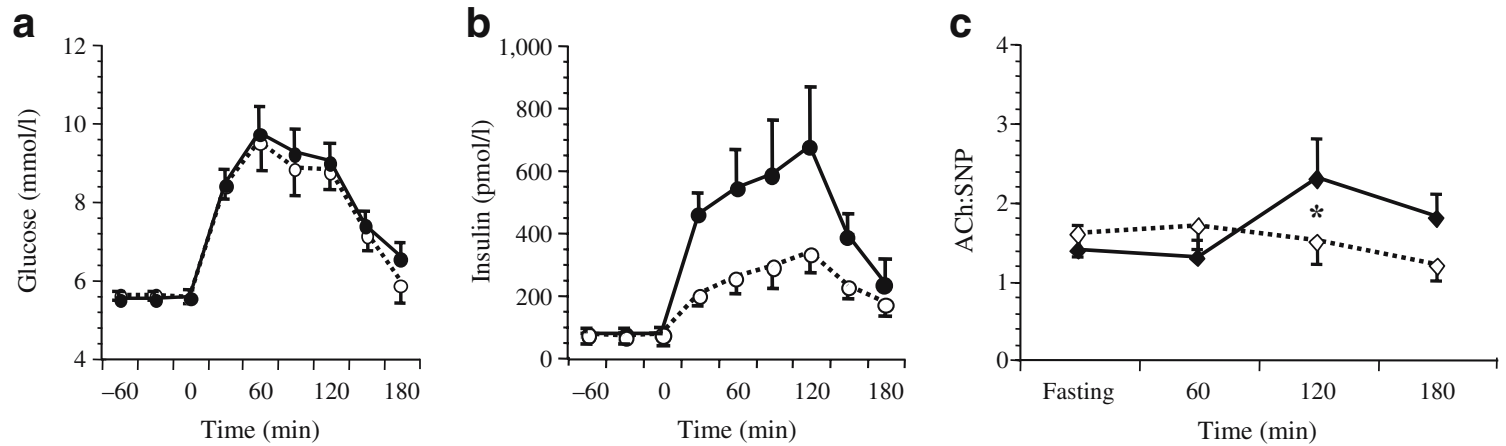

Fig. 5 a The plasma glucose and $\mathbf{b}$ insulin profiles of the isoglycaemic experiments. c Ratio of $\mathrm{ACh}$ to SNP-mediated vasodilatation in the microvasculature of IGT participants. Oral glucose

administration (black circle, solid line); isoglycaemic intravenous glucose administration (white circle, dotted line). ${ }^{*} p<0.05$

vascular tree $[39,40]$. The evidence in resistance vessels is scarce [22, 41], comes from a single laboratory and describes an inhibitory effect that is small (ACh response reduced by $20 \%$ ), only detectable for unphysiological degrees of hyperglycaemia (over $16 \mathrm{mmol} / \mathrm{l}$ ) maintained for $6 \mathrm{~h}$ and effectively prevented by a very small gradient (35 pmol/l) of hyperinsulinaemia. Other laboratories have reached opposite conclusions. Houben et al. [16], with the use of a very similar protocol (local hyperglycaemia of about $15 \mathrm{mmol} / \mathrm{l}$ ), failed to find differences in forearm microvessel and resistance vessel reactivity to ACh, SNP, norepinephrine or NO synthase inhibition, despite extending the study time to $24 \mathrm{~h}$. More recently, Reed et al. [21], using a very elegant study design, also concluded that in healthy participants, when the insulin response is prevented, neither $6 \mathrm{~h}$ of moderate hyperglycaemia $(2 \mathrm{mmol} / \mathrm{l}$ above fasting values) nor glycaemic excursions reproducing the OGTT response of IGT patients are capable of depressing the forearm vascular response to ACh or SNP. Similar findings were reported by Strey et al. [20] and Gudmundsson et al. [9], who measured the forearm vascular response before and after a mixed meal in healthy participants and before and after improvement of metabolic control in patients with type 2 diabetes. Overall, the published evidence is congruent with our data, which extend the concept of a balance between hyperglycaemia and hyperinsulinaemia to the physiological domain, across glucose tolerance status and in different vascular districts.

Among the numerous mechanisms through which in vitro hyperglycaemia impairs EDV, oxidative stress, both direct and indirect, appears to be the favoured one $[42,43]$. Our finding that oral glucose was associated with a small reduction in the antioxidant capacity of the plasma confirms what has been reported by other laboratories [44, 45]. However, as no simultaneous change in malonyldialdehyde, dROMS or lag-phase of LDL oxidisability was observed, the antioxidant response must have been sufficient to buffer the free radical excess. Alternatively, as suggested by the tendency of 8-iso- $\mathrm{PGF}_{2 \alpha}$ (a more sensitive marker of lipid membrane peroxidation) to increase in response to glucose ingestion, this specific oxidative stress might preferentially act upon targets and processes different from vascular $\mathrm{NO}$ availability. The substantial integrity of the endothelium after oral glucose is further documented by the lack of an increase in the shedding of these molecules into the circulation as commonly observed when the endothelium is activated in reaction to various harmful agents.

The observed negative impact of glucose loading on endothelium-independent vasodilatation, more evident in IGT (Fig. 2) and confirmed also in the skin microvasculature (Fig. 3), is unlikely to be due to hyperglycaemia itself, since the studies using either local or systemic hyperglycaemia have not reported this effect. It may also be unrelated to hyperinsulinaemia, since neither isolated local hyperinsulinaemia [27] nor intravenous insulin infusion [21] have been shown to affect the vascular response to SNP; however, no study to date has evaluated this response after glucose ingestion. The smaller and less persistent decline in maximal response to SNP observed when glucose was given intravenously (fasting, 651 $\pm 111 ; 60$ $\mathrm{min}, 510 \pm 88 ; 120 \mathrm{~min}, 545 \pm 90 ; 180 \mathrm{~min}, 671 \pm 121 \%$ ) vs orally (fasting, $760 \pm 127 ; 60 \mathrm{~min}, 631 \pm 89 ; 120 \mathrm{~min}, 545 \pm$ $90 ; 180 \mathrm{~min}, 549 \pm 71 \%$ ) suggests that factors related to gastro-intestinal stimulation are involved.

The baseline reduction in EDV in patients with diabetes remains unexplained. Using the forearm technique, we have previously shown that EDV is reduced in diabetic patients in proportion to their degree of insulin resistance [46]. Insulin resistance, however, does not cluster with EDV in nondiabetic normotensive participants [47] or in patients with essential hypertension [48]. Therefore, we must postulate that long-standing hyperglycaemia on its own or in interaction with insulin resistance, directly or via accumulation of AGE compromises endothelium-dependent vascular functions. A role for chronic dyslipidaemia or low-grade inflammation cannot be ruled out, but remains to be better defined. 
In conclusion, oral glucose does not impair EDV, either in resistance arteries or in the microcirculation, despite increased oxidative stress; the endogenous insulin response is quantitatively responsible for countering any inhibitory effect on vascular function even in diabetic patients, in whom EDV is compromised. Given the characteristics of the diabetic patients enrolled in this study, our conclusions cannot be extended to patients with a more severe disease, a more pronounced fasting and/or post-glucose hyperglycaemia, and/or a more blunted insulin response. Furthermore, the sample size, the experimental design and the technique employed prevented the detection of changes in resistance arteries EDV that were smaller than $20 \%$, and also of any short-duration change occurring in the first $90 \mathrm{~min}$ of the OGTT. The results of the laser Doppler technique, although consistent with plethysmography, cannot be directly extended to resistance arteries EDV and are limited to IGT participants.

Acknowledgements We wish to thank S. Burchielli and S. Pinnola for their technical assistance.

Duality of interest The authors declare that there is no duality of interest associated with this manuscript.

\section{References}

1. Ryden L, Standl E, Bartnik M et al (2007) Guidelines on diabetes, pre-diabetes, and cardiovascular diseases: executive summary: the Task Force on Diabetes and Cardiovascular Diseases of the European Society of Cardiology (ESC) and of the European Association for the Study of Diabetes (EASD). Eur Heart J 28:88136

2. Natali A, Vichi S, Landi P, Severi S, L'Abbate A, Ferrannini E (2000) Coronary atherosclerosis in Type II diabetes: angiographic findings and clinical outcome. Diabetologia 43:632-641

3. Stettler C, Allemann S, Juni P et al (2006) Glycemic control and macrovascular disease in types 1 and 2 diabetes mellitus: metaanalysis of randomized trials. Am Heart J 152:27-38

4. Ting H, Timimi F, Boles K, Creager S, Ganz P, Creager M (1996) Vitamin $\mathrm{C}$ improves endothelium-dependent vasodilatation in patients with non-insulin-dependent diabetes mellitus. J Clin Invest 97:22-28

5. Williams S (1996) Impaired nitric oxide-mediated vasodilation in patients with non-insulin-dependent diabetes mellitus. J Am Coll Cardiol 27:567-574

6. Nuttal S, Dunne F, Kendal M, Martin U (1999) Age-independent oxidative stress in elderly patients with non-insulin-dependent diabetes mellitus. Q J Med 92:33-38

7. Pickup J, Mattock M, Chusney G, Burt D (1997) NIDDM as a disease of the innate immune system: association of acute-phase reactants and interleukin-6 with metabolic syndrome X. Diabetologia 41:1241-1248

8. Ceriello A (1998) The emerging role of post-prandial hyperglycaemic spikes in the pathogenesis of diabetic complication. Diab Med 15:188-193
9. Gudmundsson G, Sinkey C, Chenard C, Stumbo P, Haynes W (2000) Resistance vessel endothelial function in healthy humans during transient post prandial hypertryglyceridemia. Am J Cardiol $85: 381-385$

10. Ceriello A, Giacomello R, Stel G et al (1995) Hyperglycemiainduced thrombin formation in diabetes. The possible role of oxidative stress. Diabetes 44:924-931

11. Rodriguez-Manas L, Angulo J, Vallejo S et al (2003) Early and intermediate Amadori glycosylation adducts, oxidative stress, and endothelial dysfunction in the streptozotocin-induced diabetic rats vasculature. Diabetologia 46:556-566

12. Tesfamariam B, Brown L, Cohen R (1991) Elevated glucose impairs endothelium-dependent relaxation by activating protein kinase C. J Clin Invest 87:1643-1648

13. Du XL, Edelstein D, Dimmeler S, Ju Q, Sui C, Brownlee M (2001) Hyperglycemia inhibits endothelial nitric oxide synthase activity by posttranslational modification at the Akt site. J Clin Invest 108:1341-1348

14. Mayhan WG (1989) Impairment of endothelium-dependent dilatation of cerebral arterioles during diabetes mellitus. Am J Physiol 256:H621-H625

15. Keegan A, Walbank H, Cotter MA, Cameron NE (1995) Chronic vitamin $\mathrm{E}$ treatment prevents defective endothelium-dependent relaxation in diabetic rat aorta. Diabetologia 38:1475-1478

16. Houben AJ, Schaper NC, De Haan CHA et al (1996) Local 24-h hyperglycemia does not affect endothelium-dependent or -independent vasoreactivity in humans. Am J Physiol 270:H2014-H2019

17. Bagg W, Whalley GA, Sathu A, Gamble G, Sharpe N, Braatvedt GD (2000) The effect of acute hyperglycaemia on brachial artery flow mediated dilatation in normal volunteers. Aust NZ J Med 30:344-350

18. Sarabi M, Fugmann A, Karlstrom B, Berne C, Lithell H, Lind L (2001) An ordinary mixed meal transiently impairs endothelium-dependent vasodilation in healthy subjects. Acta Physiol Scand 172:107-113

19. Siafarikas A, Watts K, Beye P, Jones TW, Davis EA, Green DJ (2004) Lack of effect of oral glucose loading on conduit vessel endothelial function in healthy subjects. Clin Sci (Lond) 107:191-196

20. Strey CH, Young J, Collier M, Florkowski CM, Shand BI, Scott RS (2004) The postprandial state does not impair endothelial function in women with type 2 diabetes irrespective of glycaemic control. Diabetologia 47:1838-1846

21. Reed AS, Charkoudian N, Vella A, Shah P, Rizza RA, Joyner MJ (2004) Forearm vascular control during acute hyperglycemia in healthy humans. Am J Physiol 286:E472-E480

22. Williams S, Goldfine A, Timimi F et al (1998) Acute hyperglycemia attenuates endothelium-dependent vasodilation in humans in vivo. Circulation 97:1695-1701

23. Kawano H, Motoyama T, Hirashima O et al (1999) Hyperglycemia rapidly suppresses flow-mediated endothelium-dependent vasodilation of brachial artery. Am J Coll Cardiol 34:146-153

24. Title LM, Cummings PM, Giddens K, Nassar BA (2000) Oral glucose loading acutely attenuates endothelium-dependent vasodilation in healthy adults without diabetes: an effect prevented by vitamins C and E. J Am Coll Cardiol 36:2185-2191

25. Ceriello A, Taboga C, Tonutti L et al (2002) Evidence for an independent and cumulative effect of postprandial hypertriglyceridemia and hyperglycemia on endothelial dysfunction and oxidative stress generation: effects of short- and long-term simvastatin treatment. Circulation 106:1211-1218

26. Marchesi S, Lupattelli G, Schillaci G et al (2000) Impaired flowmediated vasoactivity during post-prandial phase in young healthy men. Atherosclerosis 153:397-402

27. Taddei S, Virdis A, Mattei P, Natali A, Ferrannini E, Salvetti A (1995) Effect of insulin on acetylcholine-induced vasodilation in normal subjects and in patients with essential hypertension. Circulation 92:2911-2918 
28. Serne EH, IJzermam RG, Gans RO et al (2002) Direct evidence for insulin-induced capillary recruitment in skin of healthy subjects during physiological hyperinsulinemia. Diabetes 51: $1515-1522$

29. de Jongh RT, Clark AD, IJzermam RG, Serne EH, de Vries G, Stehouwer CD (2004) Physiological hyperinsulinaemia increases intramuscular microvascular reactive hyperaemia and vasomotion in healthy volunteers. Diabetologia 47:978-986

30. Benzie I, Strain J (1996) The ferric reducing ability of the plasma (FRAP) as a measure of "antioxidant power": the FRAP assay. Anal Biochem 15:70-76

31. Nourooz-Zadeh J, Tajaddin-Sarmadi J, Wolff SP (1994) Measurement of plasma hydroperoxide concentrations by the ferrous oxidation-xylenol orange assay in conjunction with triphenylphosphine. Anal Biochem 220:403-409

32. Baldi S, Frascerra S, Ferrannini E, Natali A (2007) LDL resistance to oxidation: effects of lipid phenotype, autologous HDL and alanine. Clin Chim Acta 379:95-100

33. Zeng G, Nystrom FH, Ravichandran LV et al (2000) Roles for insulin receptor, PI3-kinase, and Akt in insulin-signaling pathways related to production of nitric oxide in human vascular endothelial cells. Circulation 101:1539-1545

34. de Jongh RT, Serne EH, IJzermam RG, de Vries G, Stehouwer CD (2004) Impaired microvascular function in obesity: implications for obesity-associated microangiopathy, hypertension, and insulin resistance. Circulation 109:2529-2535

35. Rask-Madsen C, Ihlemann N, Krarup $T$ et al (2001) Insulin therapy improves insulin-stimulated endothelial function in patients with type 2 diabetes and ischemic heart disease. Diabetes 50:2611-2618

36. Steinberg H, Tarshoby M, Monestel R et al (1997) Elevated circulating free fatty acid levels impair endothelium-dependent vasodilation. J Clin Invest 100:1230-1239

37. Pieper G, Siebeneich W, Moore-Hilton G, Roza A (1997) Reversal by L-arginine of a dysfunctional arginine-nitric oxide pathway in endothelium of the genetic diabetic BB rat. Diabetologia 40:910-915
38. Meininger CJ, Marinos RS, Hatakeyama K et al (2000) Impaired nitric oxide production in coronary endothelial cells of the spontaneously diabetic BB rat is due to tetrahydrobiopterin deficiency. Biochem J 349:353-356

39. Arcaro G, Cretti A, Balzano S et al (2002) Insulin causes endothelial dysfunction in humans: sites and mechanisms. Circulation 105:576-582

40. Campia U, Sullivan G, Bryant MB, Waclawiw MA, Quon MJ, Panza JA (2004) Insulin impairs endothelium-dependent vasodilation independent of insulin sensitivity or lipid profile. Am J Physiol 286:H76-H82

41. Beckman J, Goldfine A, Gordon M, Creager M (2001) Ascorbate restores endothelium-dependent vasodilatation impaired by acute hyperglycemia in humans. Circulation 103:1618-1623

42. Tesfamariam B (1994) Free radicals in diabetic endothelial cell dysfunction. Free Radic Biol Med 16:383-391

43. Cosentino F, Hishikawa K, Katusic ZS, Luscher TF (1997) High glucose increases nitric oxide synthase expression and superoxide anion generation in human aortic endothelial cells. Circulation 96:25-28

44. Ceriello A, Bortolotti N, Crescentini A et al (1998) Antioxidant defenses are reduced during oral glucose tolerance test in normal and non-insulin dependent diabetic subjects. Eur J Clin Invest 28:329-334

45. Ma SW, Tomlinson B, Benzie IF (2005) A study of the effect of oral glucose loading on plasma oxidant:antioxidant balance in normal subjects. Eur J Nutr 44:250-254

46. Natali A, Toschi E, Baldeweg S et al (2006) Clustering of insulin resistance with vascular dysfunction and low-grade inflammation in type 2 diabetes. Diabetes 55:1133-1140

47. Utriainen T, Mäkimattila S, Virkamäki A, Bergholm R, YkiJärvinen H (1996) Dissociation between insulin sensitivity of glucose uptake and endothelial function in normal subjects. Diabetologia 39:1477-1482

48. Natali A, Taddei S, Quinones-Galvan A et al (1997) Insulin sensitivity, vascular reactivity, and clamp-induced vasodilatation in essential hypertension. Circulation 96:849-855 